\title{
HOLOMORPHIC LINE BUNDLES OVER DOMAINS IN COUSIN GROUPS AND THE ALGEBRAIC DIMENSION OF OT-MANIFOLDS
}

\author{
L. BATTISTI \& K. OELJEKLAUS
}

\begin{abstract}
In this paper we extend results due to Vogt on line bundles over Cousin groups to the case of domains stable by the maximal compact subgroup. This is used in the sequel to show that the algebraic dimension of OT-manifolds is zero. In the last part we establish that certain Cousin groups, in particular those arising from the construction of OT-manifolds, have finite-dimensional irregularity.
\end{abstract}

\section{INTRODUCTION}

A connected complex Lie group which admits no non-constant holomorphic functions is called a Cousin group, or also a toroidal group in the older literature. These groups are named after P. Cousin (see [3]). If $C$ is an $n$ dimensional Cousin group, it is abelian and can be realized as the quotient of $\mathbb{C}^{n}$ by a lattice $\Lambda$ of $\mathbb{C}^{n}$ of rank $n+m$, with $1 \leq m \leq n$.

It is a fact that the line bundle associated to a positive divisor on a compact Kähler manifold has a non-trivial real Chern class. In [10], C. Vogt proved the following in the non-compact context: Let $L$ be a topologically trivial line bundle on a Cousin group. Then $L$ is holomorphically trivial if and only if it admits a non-trivial holomorphic section. This result had already been proved by Cousin [3], in the special case $m=1$. His original proof was rewritten by A.T. Huckleberry and G.A. Margulis in [6], who used it to establish a theorem on hypersurfaces in quotients of semi-simple complex Lie groups, see also [2], 5].

In the first section of the present paper we generalize Vogt's result to the case of a domain $U$ in a Cousin group $C=\mathbb{C}^{n} / \Lambda$ whose inverse image in $\mathbb{C}^{n}$ is convex. This assumption implies in particular that $U$ is invariant under the action of the maximal compact subgroup of $C$.

In the next section we use the previous result to confirm the non-existence of complex hypersurfaces in OT-manifolds. In particular, the algebraic dimension of these manifolds is always zero. This result was proved in a special case by L. Ornea and M. Verbitsky in [8]. OT-manifolds were introduced in [7] and we will recall their construction later.

The last section is devoted to the observation that if the coordinates of the lattice points of a Cousin $C$ group are all algebraic integers then the irregularity $\operatorname{dim}_{\mathbb{C}} H^{1}(C, \mathcal{O})$ is finite. 
This article is organized as follows. In the first section we recall the notations of [9] and [10], and prove the following:

Theorem2.9. Let $U$ be an open subset of a Cousin group $C \cong \mathbb{C}^{n} / \Lambda$ whose inverse image $\widetilde{U}$ in $\mathbb{C}^{n}$ is a convex domain. Let $L$ be a topologically trivial holomorphic line bundle over $U$. One has $H^{0}(U, L) \neq 0$ if and only if $L$ is holomorphically trivial.

In the second section, we first recall the construction of OT-manifolds and then prove:

Theorem 3.5. Let $X$ be an OT-manifold. Then there are no complexanalytic hypersurfaces on $X$, in particular the algebraic dimension of $X$ is zero.

Before stating the result of the last section we recall:

Theorem 4.1 ([10]). Let $C=\mathbb{C}^{n} / \Lambda$ be a Cousin group. Then the following conditions are equivalent:

1. The space $H^{1}(C, \mathcal{O})$ is finite-dimensional.

2. Let $P=\left(I_{n} S\right)$ be a period basis of $\Lambda$. Then there exist constants $C>0$ and $a \geqslant 0$ such that $\left\|{ }^{t} \sigma S+{ }^{t} \tau\right\| \geqslant C \exp (-a|\sigma|)$ for all $\sigma \in \mathbb{Z}^{n} \backslash\{0\}$ and all $\tau \in \mathbb{Z}^{m}$, where $n+m$ is the rank of $\Lambda$.

3. Every line bundle over $C$ comes from a theta factor.

Applying a generalization of Liouville's theorem we obtain the following which applies in particular to Cousin groups arising in the construction of OT-manifolds:

Theorem 4.3. Let $\Lambda \subset \mathbb{C}^{n}$ be a lattice such that $C=\mathbb{C}^{n} / \Lambda$ is a Cousin group with a period basis whose coefficients are all algebraic numbers, then $C$ satisfies the equivalent conditions of theorem 4.1.

\section{Preliminaries}

Let us consider a domain $U$ of a Cousin group $C \cong \mathbb{C}^{n} / \Lambda$ whose inverse image $\widetilde{U}=\pi^{-1}(U)$ in $\mathbb{C}^{n}$ is a convex domain, where $\pi: \mathbb{C}^{n} \rightarrow \mathbb{C}^{n} / \Lambda$ is the quotient map. In particular, $U$ is invariant under the action of $\mathbb{R} \Lambda / \Lambda$, admits no non-constant holomorphic functions and $\widetilde{U}$ is invariant under the action of $\mathbb{R} \Lambda$ and is evidently Stein. We recall notations, definitions and results from [9] which adapt directly to our situation.

Definition 2.1. A map $\alpha: \Lambda \times \widetilde{U} \rightarrow \mathbb{C}^{*}$ is called a factor of automorphy if it satisfies the following properties:

a) $\alpha_{\lambda}: \widetilde{U} \rightarrow \mathbb{C}^{*}, \alpha_{\lambda}(z):=\alpha(\lambda, z)$ is holomorphic for all $\lambda \in \Lambda$, 
b) $\alpha(0, z)=1$ for all $z \in \widetilde{U}$,

c) $\alpha\left(\lambda+\lambda^{\prime}, z\right)=\alpha\left(\lambda, z+\lambda^{\prime}\right) \alpha\left(\lambda^{\prime}, z\right)$ for all $\lambda, \lambda^{\prime} \in \Lambda$ and all $z \in \widetilde{U}$.

If $\alpha$ is a factor of automorphy, for every $\lambda \in \Lambda$ there exists a holomorphic function $a_{\lambda}: \widetilde{U} \rightarrow \mathbb{C}$ unique up to an additive constant $2 i \pi k_{\lambda}$ with $k_{\lambda} \in \mathbb{Z}$, such that $\alpha(\lambda, z)=\exp \left(a_{\lambda}(z)\right)$.

Definition 2.2. A map $a: \Lambda \times \widetilde{U} \rightarrow \mathbb{C}$ is called a summand of automorphy if the following three conditions are satisfied:

a) $a(\lambda, \cdot): \widetilde{U} \rightarrow \mathbb{C}$ is holomorphic for all $\lambda \in \Lambda$,

b) $a(0, z)=0$ for all $z \in \widetilde{U}$,

c) $a\left(\lambda+\lambda^{\prime}, z\right)=a\left(\lambda, z+\lambda^{\prime}\right)+a\left(\lambda^{\prime}, z\right)$ for all $\lambda, \lambda^{\prime} \in \Lambda$ and all $z \in \widetilde{U}$.

Definition 2.3. Two factors of automorphy $\alpha$ and $\beta$ are called equivalent if there is a holomorphic function $h: \widetilde{U} \rightarrow \mathbb{C}^{*}$ satisfying

$$
\beta(\lambda, z)=h(z+\lambda) \alpha(\lambda, z) h^{-1}(z) .
$$

Two summands of automorphy are called equivalent if the induced factors of automorphy are equivalent.

Since $\widetilde{U}$ is Stein, one has the following proposition (see [9]):

Proposition 2.4. There is a bijection between equivalence classes of factors of automorphy and equivalence classes of line bundles over $U$.

We recall now two normal forms for the lattice $\Lambda$ defining a Cousin group (see [9], propositions 1 and 2):

Proposition 2.5. Let $C=\mathbb{C}^{n} / \Lambda$ be a Cousin group. Then the real rank of $\Lambda$ is $r=n+m$ with $1 \leqslant m \leqslant n$ and:

1. The Cousin group $C$ has a period basis, i.e. a basis of $\Lambda$, of the form $P=\left(I_{n} S\right)$; a lattice $\Lambda$ defined by such a matrix gives a Cousin group if and only if ${ }^{t} \sigma S \notin \mathbb{Z}^{m}$ for all $\sigma \in \mathbb{Z}^{n} \backslash\{0\}$.

2. The Cousin group $C$ has a period basis of the form $P=\left(\begin{array}{ll}0 & T \\ I_{n-m} & R\end{array}\right)$ where $T=\left(I_{m} S\right)$ is the period basis of a complex torus of dimension $m$ and $R$ is a real matrix; a lattice $\Lambda$ defined by such a matrix gives a Cousin group if and only if ${ }^{t} \sigma R \notin \mathbb{Z}^{2 m}$ for all $\sigma \in \mathbb{Z}^{n-m} \backslash\{0\}$.

2.1. Vogt's results generalized. Given a factor of automorphy on $\widetilde{U}$, one gets a line bundle $L$ on $U$ by proposition 2.4. The following proposition characterizes the topologically trivial bundles obtained that way. Its proof is the same as in the case of a Cousin group, see for instance [1], Lemma 2.1.9.

Proposition 2.6. Let $L \stackrel{p}{\rightarrow} U$ be a holomorphic line bundle given by a factor of automorphy $\alpha: \Lambda \times \widetilde{U} \rightarrow \mathbb{C}^{*}$. Then the following two conditions are equivalent:

a) the bundle $L$ is topologically trivial, 
b) there is a summand of automorphy $a: \Lambda \times \widetilde{U} \rightarrow \mathbb{C}$ such that $\alpha(\lambda, z)=$ $\exp (a(\lambda, z))$.

The previous proposition now gives a first normal form in the class of a summand of automorphy:

Proposition 2.7. Let $\Lambda \subset \mathbb{C}^{n}$ be a lattice whose period basis is given in the form $\left(I_{n} S\right)$. Then every summand of automorphy $b: \Lambda \times \widetilde{U} \rightarrow \mathbb{C}$ is equivalent to a summand of automorphy $a: \Lambda \times \widetilde{U} \rightarrow \mathbb{C}$ with the following properties:

a) $a(\sigma, z)=0$ for all $\sigma \in \mathbb{Z}^{n}$ and all $z \in \widetilde{U}$,

b) for all $\lambda \in \Lambda$, the holomorphic function $a_{\lambda}: \widetilde{U} \longrightarrow \mathbb{C}$ is

$\mathbb{Z}^{n}$-periodic.

$$
z \longmapsto a(\lambda, z)
$$

Proof: The projection $\pi: \mathbb{C}^{n} \rightarrow C$ factors through $\mathbb{C}^{n} / \mathbb{Z}^{n}$ :

$$
\mathbb{C}^{n} \stackrel{\pi_{2}}{\longrightarrow} \mathbb{C}^{n} / \mathbb{Z}^{n} \stackrel{\pi_{1}}{\longrightarrow} C
$$

with $\pi=\pi_{1} \circ \pi_{2}$. The image of $\widetilde{U}$ by $\pi_{2}$ in $\mathbb{C}^{n} / \mathbb{Z}^{n} \cong\left(\mathbb{C}^{*}\right)^{n}$ is Stein by Grauert-Docquier's theorem. Let $L$ be the line bundle defined by the summand of automorphy $b$. By the previous proposition, $L$ is topologically trivial, so is $\pi_{1}^{*} L$. Because every topologically trivial line bundle over a Stein manifold is holomorphically trivial, one obtains a trivialization of $\pi^{*} L$ by taking the pull-back of a trivialization of $\pi_{1}^{*} L$. The summand of automorphy $a: \Lambda \times \widetilde{U} \rightarrow \mathbb{C}$ given by this trivialization is equivalent to $b$ in the sense of definition 2.3 and it satisfies condition $a$ ). Condition $b$ ) is a consequence of both $a$ ) and the definition of a summand of automorphy.

One also has a second normal form for summands of automorphy:

Proposition 2.8. Let $\Lambda \subset \mathbb{C}^{n}$ be a lattice whose basis is given under the form

$$
P=\left(\begin{array}{ll}
0 & T \\
I_{n-m} & R
\end{array}\right)
$$

such that $C=\mathbb{C}^{n} / \Lambda$ is a Cousin group. Then every summand of automorphy $b: \Lambda \times \widetilde{U} \rightarrow \mathbb{C}$ is equivalent to a summand of automorphy $a: \Lambda \times \widetilde{U} \rightarrow \mathbb{C}$ with the following properties:

a) $a(\lambda, z)=a\left(\lambda, z_{m+1}, \ldots, z_{n}\right)$ for all $\lambda \in \Lambda$ and all $z \in \widetilde{U}$,

b) $a(\lambda, z)=0$ for all $\lambda \in\left(\begin{array}{c}0 \\ \mathbb{Z}^{n-m}\end{array}\right)$ and all $z \in \widetilde{U}$,

c) for all $\lambda \in \Lambda$, the holomorphic function $a_{\lambda}: \widetilde{U} \longrightarrow \mathbb{C}$ is $\left(\begin{array}{c}0 \\ \mathbb{Z}^{n-m}\end{array}\right)$-periodic. 

here.

The proof given in [9] readily adapts to our case so we will not repeat it

Now, we state the main result of this section:

Theorem 2.9. Let $U$ be an open subset of a Cousin group $C \cong \mathbb{C}^{n} / \Lambda$ whose inverse image $\widetilde{U}$ in $\mathbb{C}^{n}$ is a convex domain. Let $L$ be a topologically trivial holomorphic line bundle over $U$. One has $H^{0}(U, L) \neq 0$ if and only if $L$ is holomorphically trivial.

Proof: The sufficient condition is clear so we prove the necessary condition. We choose the second normal form of the lattice of proposition 2.5. Since $L$ is topologically trivial, proposition 2.6 implies that it is given by a summand of automorphy $a: \Lambda \times \widetilde{U} \rightarrow \mathbb{C}$. By using the normal form of proposition 2.8 we may assume that:

- $a(\lambda, z)=a\left(\lambda, z_{m+1}, \ldots, z_{n}\right)$ for all $\lambda \in \Lambda$ and

$\cdot a_{\lambda}: \widetilde{U} \rightarrow \mathbb{C}, z \mapsto a(\lambda, z)$ is $\left(\begin{array}{c}0 \\ \mathbb{Z}^{n-m}\end{array}\right)$-periodic for all $\lambda$.

Now consider the Fourier series of $a_{\lambda}$ :

$$
a_{\lambda}(z)=\sum_{\sigma \in \mathbb{Z}^{n-m}} a_{\lambda, \sigma} e^{2 i \pi^{t} \sigma\left(z_{m+1}, \ldots, z_{n}\right)} .
$$

Since $a$ is a summand of automorphy, we have:

$$
a_{\lambda}\left(z+\lambda^{\prime}\right)+a_{\lambda^{\prime}}(z)=a_{\lambda^{\prime}}(z+\lambda)+a_{\lambda}(z)
$$

hence:

$$
a_{\lambda, \sigma}\left(1-e^{2 i \pi^{t} \sigma \lambda_{2}^{\prime}}\right)=a_{\lambda^{\prime}, \sigma}\left(1-e^{2 i \pi^{t} \sigma \lambda_{2}}\right)
$$

for $\lambda, \lambda^{\prime} \in \Lambda$ (where $\lambda_{2}$ [resp. $\lambda_{2}^{\prime}$ ] is the $(n-m)$-tuple consisting of the $n-m$ last coordinates of $\lambda$ [resp. $\left.\left.\lambda^{\prime}\right]\right)$ and $z \in \widetilde{U}$.

We want to extend the map $a$ to $\mathbb{R} \Lambda \times \widetilde{U}$ such that

$$
a\left(x+x^{\prime}, z\right)=a\left(x, z+x^{\prime}\right)+a\left(x^{\prime}, z\right) \text { for all } x, x^{\prime} \in \mathbb{R} \Lambda, z \in \mathbb{C}^{n} .
$$

First, we define $a(x, z)$ for an element $x$ of $\mathbb{R} \Lambda$ of the form $x=r \lambda$ by:

$$
a(x, z):=\sum_{\sigma \in \mathbb{Z}^{n-m}} a_{\lambda, \sigma} C(\sigma, r, \lambda) e^{2 i \pi^{t} \sigma\left(z_{m+1}, \ldots, z_{n}\right)},
$$

where

$$
C(\sigma, r, \lambda)=\left\{\begin{array}{cl}
\frac{1-e^{2 i \pi r^{t} \sigma \lambda_{2}}}{1-e^{2 i \pi^{t} \sigma \lambda_{2}}} & \text { if }{ }^{t} \sigma \lambda_{2} \notin \mathbb{Z} \\
\lim _{u \rightarrow 0} \frac{1-e^{i r u}}{1-e^{i u}}=r & \text { if }{ }^{t} \sigma \lambda_{2} \in \mathbb{Z} .
\end{array}\right.
$$

The Fourier series defining $a(x, z)$ is convergent since for any fixed real $r$, the factors $C(\sigma, r, \lambda)$ are uniformly bounded by $r$.

Now for an element of $\mathbb{R} \Lambda$ of the form $x=\sum_{j=1}^{n+m} r_{j} \lambda_{j}$ we define the differentiable function $a(x, z)$ everywhere on $\mathbb{R} \Lambda \times \widetilde{U}$ using equation (1). 
Consider a section $s$ of $L$. We shall prove that if $s$ vanishes at one point, then it is constant equal to zero. This section corresponds to a holomorphic function $f: \widetilde{U} \rightarrow \mathbb{C}$, satisfying

$$
f(z+\lambda)=e^{a(\lambda, z)} f(z)
$$

for all $\lambda \in \Lambda$ and all $z \in \widetilde{U}$.

Let $z_{0} \in \widetilde{U}$, consider the function:

$$
\begin{aligned}
t_{z_{0}}: \mathbb{R} \Lambda & \longrightarrow \mathbb{C} \\
x & \longmapsto f\left(z_{0}+x\right) e^{-a\left(x, z_{0}\right)} .
\end{aligned}
$$

Then, for all $\lambda \in \Lambda$ and all $x \in \mathbb{R} \Lambda$, one has:

$$
\begin{aligned}
t_{z_{0}}(x+\lambda) & =f\left(z_{0}+x+\lambda\right) e^{-a\left(x+\lambda, z_{0}\right)} \\
& =f\left(z_{0}+x\right) e^{a\left(\lambda, z_{0}+x\right)-a\left(x+\lambda, z_{0}\right)} \\
& =f\left(z_{0}+x\right) e^{-a\left(x, z_{0}\right)} \\
& =t_{z_{0}}(x) .
\end{aligned}
$$

Hence $t_{z_{0}}$ is a $\Lambda$-periodic function and therefore bounded by a constant $C_{z_{0}}$. This implies that for all $x \in \mathbb{R} \Lambda$,

$$
\left|f\left(z_{0}+x\right)\right| \leqslant C_{z_{0}}\left|e^{a\left(x, z_{0}\right)}\right|=C_{z_{0}} e^{\Re a\left(x, z_{0}\right)} .
$$

Let $\mathbb{C}_{\Lambda}^{m}$ be the maximal complex subspace of $\mathbb{R} \Lambda$. We need that the function $x \mapsto \Re\left(a\left(x, z_{0}\right)\right)$ is bounded. In order to satisfy this, we will slightly modify the map $a$. There exists a unique linear map $g_{z_{0}}: \mathbb{C}_{\Lambda}^{m} \rightarrow \mathbb{C}$ such that $\Re\left(g_{z_{0}}\left(\lambda_{1}\right)\right)=\Re\left(a\left(\lambda_{1}, z_{0}\right)\right)$ for all $\lambda \in \Lambda$. by:

We then consider the summand of automorphy $\widetilde{a}_{z_{0}}: \Lambda \times \widetilde{U} \rightarrow \mathbb{C}$ defined

$$
\widetilde{a}_{z_{0}}(\lambda, z)=a(\lambda, z)-g_{z_{0}}(z+\lambda)+g_{z_{0}}(z)=a(\lambda, z)-g_{z_{0}}(\lambda)
$$

it defines the same line bundle as $a$. Hence, up to exchanging $a$ with $\widetilde{a}_{z_{0}}$, one can now assume that $\Re\left(a\left(\lambda_{1}, z_{0}\right)\right)=0$ for all $\lambda \in \Lambda$. For $x \in \mathbb{C}_{\Lambda}^{m}$ and $\lambda \in P$, one has:

$$
\begin{aligned}
a\left(x+\lambda_{1}, z_{0}\right) & =a\left(x, z_{0}+\lambda_{1}\right)+a\left(\lambda_{1}, z_{0}\right) \\
& =a\left(x, z_{0}\right)+a\left(\lambda_{1}, z_{0}\right)
\end{aligned}
$$

(since by assumption on $a$, in its second argument it only depends on the last $n-m$ coordinates), so the restriction of $\Re a$ to $\mathbb{C}_{\Lambda}^{m} \times\left\{z_{0}\right\}$ is $\widetilde{T} . \mathbb{Z}^{2 m}$ periodic therefore bounded. Consequently, the restriction of $f\left(z_{0}+\cdot\right)$ to $\mathbb{C}_{\Lambda}^{m}$ is a bounded holomorphic function, so it is constant and this holds for all $z_{0} \in \widetilde{U}$. This lets us see that $f$ does not depend on the first $m$ coordinates; we can see $f$ as a holomorphic function over $\widetilde{U}_{2}=\left\{z_{2} \mid z \in \widetilde{U}\right\}$. The zeros of $f$ are $\left(I_{n-m} R\right)$-invariant, because of condition (2). Since $\mathbb{C}^{n} / \Lambda$ is a Cousin group, there is no $\sigma \in \mathbb{Z}^{n-m} \backslash\{0\}$ with ${ }^{t} \sigma R \in \mathbb{Z}^{2 m}$ so the group generated by $\left(I_{n-m} R\right)$ is dense in $\mathbb{R}^{n-m}$. It is a consequence that if $f(w)=0$ for some $w \in \widetilde{U}_{2}$, then $f$ vanishes on $w+\mathbb{R}^{n-m}$ and also on the intersection of $\widetilde{U}_{2}$ and the complex linear subspace $\mathbb{C}^{n-m}$ generated by $w+\mathbb{R}^{n-m}$, i.e. 
$\widetilde{U}_{2} \cap \mathbb{C}^{n-m}=\widetilde{U}_{2}$. Finally, if $s$ is a non-trivial section of $L$, it never vanishes and $L$ is holomorphically trivial.

Now we give a reformulation of the previous proposition which will be used in the next section:

Lemma 2.10. Let $U$ be an open set of a Cousin group $C \cong \mathbb{C}^{n} / \Lambda$ whose inverse image $\widetilde{U}$ in $\mathbb{C}^{n}$ is a convex domain and let $D$ be a positive divisor of $U$. Let $L$ be the line bundle over $U$ associated to $D$. Then $L$ is not topologically trivial, i.e. its first Chern class $c_{1}(L) \in H^{2}(U, \mathbb{Z})$ is not zero.

Proof: Assume that $L$ is topologically trivial. By theorem 2.9, since it admits a (non trivial) section $s_{1}$ given by $D$, it is holomorphically trivial. This means that it has a holomorphic section $s_{2}$ which never vanishes. The quotient $s_{1} / s_{2}$ is a non-constant holomorphic function on $U$ (since $s_{1}$ vanishes on $D$ and not on the complement of $D$ ), a contradiction. One can also notice that $s_{1}$ vanishes on $D$ so it is identically zero by the proof of the previous theorem, which leads to a contradiction too.

\section{Hypersurfaces on OT-MANifolds}

The goal of this section is to prove that the algebraic dimension of an OTmanifold is zero. We first briefly recall the construction of OT-manifolds.

3.1. Construction of OT-manifolds. Let $K$ be a number field of degree $n$ over $\mathbb{Q}$; call $s$ (resp. 2t) the number of real (resp. complex) embeddings of $K$, so that $n=s+2 t$. For the construction, one needs to assume that the integers $s$ and $t$ are non-zero.

Then $\sigma\left(\mathcal{O}_{K}\right)$ is a lattice of rank $n$ in $\mathbb{C}^{m}$ (where we set $m:=s+t$ ) hence we have a properly discontinuous action of $\sigma\left(\mathcal{O}_{K}\right)$ on $\mathbb{C}^{m}$. We also define $l: \mathcal{O}_{K}^{*,+} \rightarrow \mathbb{R}^{m}$ by

$$
l(a)=\left(\log \left|\sigma_{1}(a)\right|, \ldots, \log \left|\sigma_{s}(a)\right|, 2 \log \left|\sigma_{s+1}(a)\right|, \ldots, 2 \log \left|\sigma_{m}(a)\right|\right) .
$$

By Dirichlet's units theorem, $l\left(\mathcal{O}_{K}^{*,+}\right)$ is a lattice in the vector space $L:=$ $\left\{x \in \mathbb{R}^{m} \mid \sum_{i=1}^{m} x_{i}=0\right\}$. The projection $p r: L \rightarrow \mathbb{R}^{s}$ given by the $s$ first coordinates is surjective so there are subgroups $A$ of rank $s$ of $\mathcal{O}_{K}^{*++}$ such that $\operatorname{pr}(l(A))$ is a lattice of rank $s$ of $\mathbb{R}^{s}$. Such an $A$ is called admissible. We then look at the quotient $X:=X(K, A)=\left(\mathbb{H}^{s} \times \mathbb{C}^{t}\right) /\left(A \ltimes \mathcal{O}_{K}\right)$; it is a complex compact manifold of dimension $m$. This manifold is called an Oeljeklaus-Toma manifold, or OT-manifold.

We know ([7], lemma 2.4) that $\mathbb{C}^{m} / \sigma\left(\mathcal{O}_{K}\right)$ admits no non-constant holomorphic function. In other words, the complex Lie group $C:=\mathbb{C}^{m} / \sigma\left(\mathcal{O}_{K}\right)$ is a Cousin group.

We recall two lemmas and a definition (see [7]) for further use: 
Lemma 3.1. Let $A$ be a subgroup of $\mathcal{O}_{K}^{*,+}$ which is not contained in $\mathbb{Z}$. Then the two following conditions are equivalent:

1. The action of $A$ on $\mathcal{O}_{K}$ admits a (non-trivial) proper invariant submodule of lower rank.

2. There exists a proper intermediate field extension $\mathbb{Q} \subset K^{\prime} \subset K$ with $A \subset \mathcal{O}_{K^{\prime}}^{*++}$.

Definition 3.2. We say that $X(K, A)$ is of simple type if $A$ does not satisfy one of the equivalent conditions of the previous lemma.

Lemma 3.3. Let $\mathbb{Q} \subset K^{\prime} \subset K$ an intermediate field extension with $A \subset$ $\mathcal{O}_{K^{\prime}}^{*++}$ an admissible subgroup for $K$. Let $s^{\prime}, 2 t^{\prime}$ be the number of real and complex embeddings of $K^{\prime}$ respectively. Then $s=s^{\prime}, t^{\prime}>0$ and $A$ is admissible for $K^{\prime}$.

For more details, see [7].

3.2. Algebraic dimension. We shall first consider the case of an OTmanifold of simple type.

Proposition 3.4. Let $X$ be an OT-manifold of simple type. Then it admits no irreducible divisor; in particular, the algebraic dimension of $X$ is zero.

Proof: Assume that $D$ is an irreducible divisor of $X$ of multiplicity 1 . We consider $X=X(K, A)$ as the quotient of $U:=\left(\mathbb{H}^{s} \times \mathbb{C}^{t}\right) / \sigma\left(\mathcal{O}_{K}\right)$ (which is diffeomorphic to $\left.\left(\mathbb{R}_{>0}\right)^{s} \times\left(\mathbb{S}^{1}\right)^{n}\right)$ by $A \cong \mathbb{Z}^{s}$. One has the following commutative diagram:

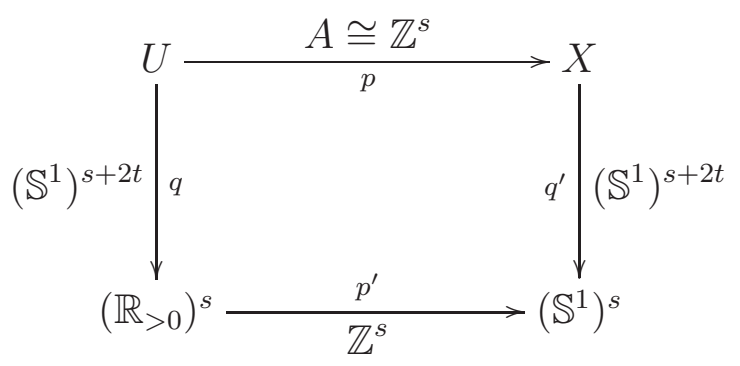

Because $X$ is of simple type, one has $\operatorname{dim} H^{2}(X)=\left(\begin{array}{l}s \\ 2\end{array}\right)=\operatorname{dim} H^{2}\left(\left(\mathbb{S}^{1}\right)^{s}\right)$ by proposition 2.3 of [7]. Thus the first Chern class $c_{1}(L(D))$ of the line bundle $L(D)$ over $X$ associated to $D$ is the pull-back by $q^{\prime}$ of an element $\omega \in H^{2}\left(\left(\mathbb{S}^{1}\right)^{s}, \mathbb{Z}\right)$. The fact that the $\operatorname{map}\left(q^{\prime}\right)^{*}: H^{2}\left(\left(\mathbb{S}^{1}\right)^{s}, \mathbb{Z}\right) \rightarrow H^{2}(X, \mathbb{Z})$ is injective is established in the proof of proposition 2.3 of [7]. The commutativity of diagram (44) implies that the pull-back $\widetilde{\omega}$ over $U$ of $\omega$ by $q^{\prime} \circ p$ is the same as the pull-back by $p^{\prime} \circ q$ of $\omega$. Furthermore, one has $\left(p^{\prime}\right)^{*}(\omega)=0$ since $H^{2}\left(\left(\mathbb{R}_{>0}\right)^{s}, \mathbb{Z}\right)=0$. Hence $\widetilde{\omega}=0$. This is a contradiction to lemma 2.10, since $\widetilde{\omega}=c_{1}(\widetilde{L}(D))$ where $\widetilde{L}(D)$ is the bundle associated to the divisor $p^{-1}(D)$ of $U$.

Now we can omit the hypothesis that $X$ is of simple type: 
Theorem 3.5. Let $X$ be an OT-manifold. Then there are no complexanalytic hypersurfaces on $X$, in particular the algebraic dimension of $X$ is zero.

Proof: By proposition 3.4, we can assume that $X$ is not of simple type. Consequently there exists a field extension $\mathbb{Q} \subset K^{\prime} \subset K$ with $A \subset \mathcal{O}_{K^{\prime}}^{*++}$ and we call $s^{\prime}$ (respectively, $2 t^{\prime}$ ) the number of real (respectively, complex) embeddings of $K^{\prime}$. Since $A$ is admissible for $K$, we have $s=s^{\prime}$ and $A$ is admissible for $K^{\prime}$ (see lemma 3.3). Without loss of generality, we can suppose that $K^{\prime}$ is the smallest subfield of $K$ such that $A \subset \mathcal{O}_{K^{\prime}}^{*,+}$, i.e. $X\left(K^{\prime}, A\right)$ is of simple type. Call $\sigma_{1}, \ldots, \sigma_{s}, \sigma_{s+1}, \ldots, \sigma_{s+t}, \bar{\sigma}_{s+1}, \ldots, \bar{\sigma}_{s+t}$ the $s+2 t$ embeddings of $K$ and $\sigma_{1}^{\prime}, \ldots, \sigma_{s}^{\prime}, \sigma_{s+1}^{\prime}, \ldots, \sigma_{s+t^{\prime}}^{\prime}, \bar{\sigma}_{s+1}^{\prime}, \ldots, \bar{\sigma}_{s+t^{\prime}}^{\prime}$ the $s+2 t^{\prime}$ embeddings of $K^{\prime}$.

As before, we note

$$
\begin{aligned}
\sigma: \mathcal{O}_{K} & \longrightarrow \mathbb{C}^{s+t} \\
a & \longmapsto\left(\sigma_{1}(a), \ldots, \sigma_{s+t}(a)\right) .
\end{aligned}
$$

We look at the complex linear subspace $V_{K^{\prime}}$ of $\mathbb{C}^{s+t}$ spanned by $\sigma\left(\mathcal{O}_{K^{\prime}}\right)$. We denote by $C$ (respectively, $C^{\prime}$ ) the Cousin group $\mathbb{C}^{s+t} / \sigma\left(\mathcal{O}_{K}\right)$ (respectively, $\left.V_{K^{\prime}} / \sigma\left(\mathcal{O}_{K^{\prime}}\right)\right)$. The group $C^{\prime}$ is a closed complex Lie subgroup of $C$. We study the two open sets $U:=\left(\mathbb{H}^{s} \times \mathbb{C}^{t}\right) / \sigma\left(\mathcal{O}_{K}\right) \subset C$ and $U^{\prime}:=$ $\left(\left(\mathbb{H}^{s} \times \mathbb{C}^{t}\right) \cap V_{K^{\prime}}\right) / \sigma\left(\mathcal{O}_{K^{\prime}}\right) \subset C^{\prime}$. The quotients $U / A$ and $U^{\prime} / A$ are OTmanifolds that we call $X$ and $X^{\prime}$ respectively; moreover, $X^{\prime}$ is a compact complex submanifold of $X$.

We consider the following commutative diagram:

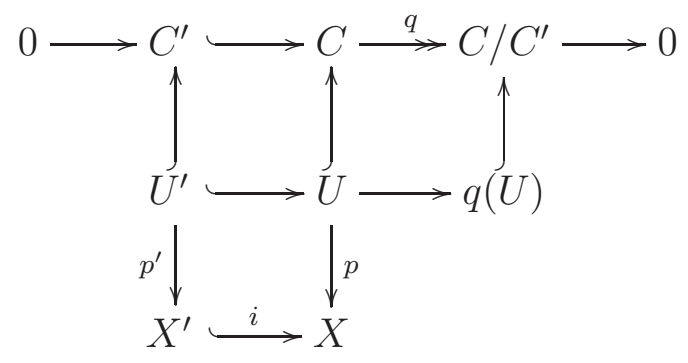

Let $D$ be a irreducible divisor of multiplicity 1 of $X$ and let $L(D)$ be the associated holomorphic line bundle. By restriction we get a bundle $L^{\prime}:=i^{*} L(D)$ over $X^{\prime}$.

Since $X^{\prime}$ is of simple type, the line bundle $\left(p^{\prime}\right)^{*}\left(L^{\prime}\right)$ over $U^{\prime}$ is topologically trivial, see the proof of proposition 3.4. The maximal complex subgroup $H^{\prime} \cong \mathbb{C}^{t^{\prime}}$ of the maximal compact torus $T^{\prime} \cong\left(\mathbb{S}^{1}\right)^{s+2 t^{\prime}}$ of $C^{\prime}$ is a subgroup of the maximal complex subgroup $H \cong \mathbb{C}^{t}$ of the maximal compact torus $T \cong\left(\mathbb{S}^{1}\right)^{s+2 t}$ of $C$. We denote by $B \cong \mathbb{C}^{t-t^{\prime}}$ a connected complex subgroup of $T$ such that $B \times H^{\prime} \cong H$. Since $B$ is a subgroup of $T$ it acts on $U$ and this action is transitive on the leaves of the $U^{\prime}$-foliation of $U$ induced by the $C^{\prime}$-foliation of $C$. The induced action of $B$ on $H^{2}(U, \mathbb{Z})$ is trivial since 
$H^{2}(U, \mathbb{Z})$ is discrete and $B$ is connected, i.e. we have $b^{*} c_{1}(L)=c_{1}(L)$ for every line bundle $L$ above $U$ and all $b \in B$.

The inverse image $\widetilde{D}:=p^{-1}(D)$ is a divisor of $U$. We shall now prove that the divisor $\widetilde{D}$ is saturated by the leaves of the $U^{\prime}$-foliation of $U$.

We have

$$
c_{1}(L(\widetilde{D}))=p^{*}\left(c_{1}(L(D))\right) \in H^{2}(U, \mathbb{Z})
$$

Since the diagram (5) commutes, we have $c_{1}\left(\left.L(\widetilde{D})\right|_{U^{\prime}}\right)=\left.c_{1}(L(\widetilde{D}))\right|_{U^{\prime}}=$ $c_{1}\left(p^{*} L^{\prime}\right)=0=\left.c_{1}\left(b^{*} L(\widetilde{D})\right)\right|_{U^{\prime}}$. There are three possible cases for the intersection $b(\widetilde{D}) \cap U^{\prime}$. Either this intersection is $U^{\prime}$, or it is empty (these two cases fit our purpose), or it is a divisor of $U^{\prime}$. The last case can not occur since $\left.c_{1}\left(b^{*} L(\widetilde{D})\right)\right|_{U^{\prime}}$ would be non-zero by lemma 2.10, which is a contradiction to (6) . Hence $\widetilde{D}$ is saturated by the leaves of the $U^{\prime}$-foliation of $U$.

Now, $\widetilde{D}$ induces a divisor $q(\widetilde{D})$ of $q(U)$. One has $q(U)=C / C^{\prime}$ because the leaves of the $U^{\prime}$-foliation of $U$ are in bijection with those of the $C^{\prime}$ foliation of $C$. Hence, $q(\widetilde{D})$ lifts as a divisor in $C$, which is stable under the action of the group $A \ltimes C^{\prime}$. We still denote by $\widetilde{D}$ this divisor.

Since the group $\mathcal{O}_{K}^{*,+}$ is abelian, the action of $\mathcal{O}_{K}^{*,+}$ on $U$ induces an action of $\mathcal{O}_{K}^{*,+}$ on $X$. Let $\eta \in \mathcal{O}_{K}^{*,+}$, we have $\widetilde{\eta D}=\eta \widetilde{D}$. We use the $C^{\prime}$-invariance of $\widetilde{\eta D}$ to write $\eta \widetilde{D}+C^{\prime}=\eta \widetilde{D}$ hence $\widetilde{D}$ is both $C^{\prime}$-invariant and $\eta^{-1} C^{\prime}$-invariant.

Let $J$ be the smallest connected complex subgroup of $C$ containing $C^{\prime}$ and $\eta C^{\prime}$ for all $\eta \in \mathcal{O}_{K}^{*,+}$. If $J$ is a proper subgroup of $C$, its maximal compact subgroup is defined by a sublattice of $\mathcal{O}_{K}$ which is stable under the action of $\mathcal{O}_{K}^{*,+}$. This is impossible because there is no sublattice of $\mathcal{O}_{K}$ stable by $\mathcal{O}_{K}^{*,+}$. Indeed, if it were the case, since $\mathcal{O}_{K}^{*,+}$ contains a primitive element of $K$, this element should have a minimal polynomial with degree strictly smaller than the one of $K$ and this is impossible. Finally, $J=C$ and $\widetilde{D}$ is invariant by $C$, which is a contradiction. Hence, $X$ admits no divisor and its algebraic dimension is zero.

\section{A special Class of Cousin groups}

4.1. Introduction. In [9], Vogt exhibits a special class of Cousin groups by prooving:

Theorem 4.1. Let $C=\mathbb{C}^{n} / \Lambda$ be a Cousin group. Then the following conditions are equivalent:

1. The space $H^{1}(C, \mathcal{O})$ is finite-dimensional.

2. Let $P=\left(I_{n} S\right)$ be a period basis of $\Lambda$. Then there exist constants $\mathcal{C}>0$ and $a \geqslant 0$ such that $\left\|{ }^{t} \sigma S+{ }^{t} \tau\right\| \geqslant \mathcal{C} \exp (-a|\sigma|)$ for all $\sigma \in \mathbb{Z}^{n} \backslash\{0\}$ and all $\tau \in \mathbb{Z}^{m}$, where $n+m$ is the rank of $\Lambda$.

3. Every line bundle over $C$ comes from a theta factor.

\footnotetext{
${ }^{1}$ The theorem given in [9], p. 208 has 8 equivalent assertions, here we only recall three of them.
} 
Let $\alpha$ be a real irrational algebraic number. In his paper, Vogt gives the following example of a lattice in $\mathbb{C}^{2}$ given by $P=\left(\begin{array}{lll}0 & 1 & i \\ 1 & \alpha & 0\end{array}\right)$ which defines a Cousin group satisfying condition $n^{\circ} 2$. Here we give a general theorem in this setting which in particular applies to all Cousin groups appearing in the construction of OT-manifolds.

We shall use the following generalization of Liouville's theorem which can be found in [4] (theorem 1.5, page 27):

Theorem 4.2. Let $\alpha_{1}, \ldots, \alpha_{m}$ be algebraic numbers, of respective degrees $n_{k}$, with $\operatorname{deg} \mathbb{Q}\left(\alpha_{1}, \ldots, \alpha_{m}\right)=n$, and let

$$
P\left(z_{1}, \ldots, z_{m}\right)=\sum_{k_{1}=0}^{N_{1}} \cdots \sum_{k_{m}=0}^{N_{m}} a_{k_{1}, \ldots, k_{m}} z_{1}^{k_{1}} \cdots z_{m}^{k_{m}} \in \mathbb{Z}\left[z_{1}, \ldots, z_{m}\right] .
$$

If $P\left(\alpha_{1}, \ldots, \alpha_{m}\right)$ is non-zero, then one has the inequality

$$
\left|P\left(\alpha_{1}, \ldots, \alpha_{m}\right)\right| \geqslant L(P)^{1-\delta n} \prod_{k=1}^{m} L\left(\alpha_{k}\right)^{-\delta N_{k} n / n_{k}},
$$

with $\delta=1$ if all the $\alpha_{i}$ are real, $\delta=1 / 2$ otherwise and where $L(P)$ is the sum of the absolute values of the coefficients of $P$ (and $L(\alpha)$ is the quantity $L(\mu)$, with $\mu$ being the minimal polynomial of $\alpha$ ).

4.2. A class of Cousin groups satisfying condition $\mathbf{n}^{\circ} \mathbf{2}$. We shall show:

Theorem 4.3. Let $\Lambda \subset \mathbb{C}^{n}$ be a lattice such that $C=\mathbb{C}^{n} / \Lambda$ is a Cousin group with a period basis whose coefficients are all algebraic numbers, then $C$ satisfies the equivalent conditions of theorem 4.1 .

Proof: Let us write a period basis of $\Lambda$ as a matrix

$$
\left(I_{n} S\right)
$$

where $I_{n}$ is the identity matrix of size $n$ and $S=\left(s_{i, j}\right)$ is a matrix with $n$ rows and $m$ columns whose coefficients are algebraic numbers over $\mathbb{Q}$.

In order the check that the second condition of theorem 4.1 is satisfied, we have to verify that there exist constants $\mathcal{C}>0$ and $a \geqslant 0$ such that for all $\mu \in \mathbb{Z}^{n} \backslash\{0\}$ and all $\nu \in \mathbb{Z}^{m}$, one has

$$
\left\|{ }^{t} \mu S+{ }^{t} \nu\right\| \geqslant \mathcal{C} \exp (-a|\mu|)
$$

It is enough to prove this inequality for any non-zero coordinate of the vector ${ }^{t} \mu S+{ }^{t} \nu$ and then consider the infinity norm of this vector. Denote ${ }^{t} \mu=\left(\mu_{1}, \ldots, \mu_{n}\right)$ and ${ }^{t} \nu=\left(\nu_{1}, \ldots, \nu_{m}\right)$. Then the $k$-th coordinate of the vector ${ }^{t} \mu S+{ }^{t} \nu$ (for $\left.k \in\{1, \ldots, m\}\right)$ is

$$
\mu_{1} s_{1, k}+\cdots+\mu_{n} s_{n, k}+\nu_{k} .
$$

We now distinguish the two following cases: either we have the inequality $\left|\nu_{k}\right| \leqslant 2\left|\mu_{1} s_{1, k}+\cdots+\mu_{n} s_{n, k}\right|$, or its converse holds. 
Suppose first $\left|\nu_{k}\right| \leqslant 2\left|\mu_{1} s_{1, k}+\cdots+\mu_{n} s_{n, k}\right|$. By theorem 4.2, we have

$$
\left|\mu_{1} s_{1, k}+\cdots+\mu_{n} s_{n, k}+\nu_{k}\right| \geqslant \mathcal{C}\left(\left|\mu_{1}\right|+\cdots+\left|\mu_{n}\right|+\left|\nu_{k}\right|\right)^{p},
$$

where $\mathcal{C}>0$ and $p<0$ are constants independent of $\mu$ and $\nu$. Recall here that we assumed that $\mu_{1} s_{1, k}+\cdots+\mu_{n} s_{n, k}+\nu_{k}$ is non-zero. By assumption on $\nu_{k}$, we have

$$
\begin{aligned}
\left(\left|\mu_{1}\right|+\cdots+\left|\mu_{n}\right|+\left|\nu_{k}\right|\right)^{p} & \geqslant\left(\left|\mu_{1}\right|+\cdots+\left|\mu_{n}\right|+2\left|\mu_{1} s_{1, k}+\cdots+\mu_{n} s_{n, k}\right|\right)^{p} \\
& \geqslant \mathcal{C}^{\prime}\left(\left|\mu_{1}\right|+\cdots+\left|\mu_{n}\right|\right)^{p} \\
& \geqslant \mathcal{C}^{\prime} \exp \left(-|p|\left(\left|\mu_{1}\right|+\cdots+\left|\mu_{n}\right|\right)\right),
\end{aligned}
$$

with $\mathcal{C}^{\prime}$ independent of $\mu$ and $\nu$.

Now suppose that $\left|\nu_{k}\right|>2\left|\mu_{1} s_{1, k}+\cdots+\mu_{n} s_{n, k}\right|$. We write the reverse triangle inequality:

$$
\begin{aligned}
\left|\mu_{1} s_{1, k}+\cdots+\mu_{n} s_{n, k}+\nu_{k}\right| & \geqslant|| \nu_{k}|-| \mu_{1} s_{1, k}+\cdots+\mu_{n} s_{n, k}|| \\
& =\left|\nu_{k}\right|-\left|\mu_{1} s_{1, k}+\cdots+\mu_{n} s_{n, k}\right| \\
& \geqslant\left|\mu_{1} s_{1, k}+\cdots+\mu_{n} s_{n, k}\right| .
\end{aligned}
$$

If $\mu_{1} s_{1, k}+\cdots+\mu_{n} s_{n, k}$ vanishes, we have $\left|\nu_{k}\right| \geqslant 1$ and the result is obtained; otherwise, we can again use theorem 4.2 .

$$
\begin{aligned}
\left|\mu_{1} s_{1, k}+\cdots+\mu_{n} s_{n, k}\right| & \geqslant \mathcal{C}^{\prime \prime}\left(\left|\mu_{1}\right|+\cdots+\left|\mu_{n}\right|\right)^{q} \\
& \geqslant \mathcal{C}^{\prime \prime} \exp \left(-|q|\left(\left|\mu_{1}\right|+\cdots\left|\mu_{n}\right|\right)\right)
\end{aligned}
$$

where $\mathcal{C}^{\prime \prime}>0$ and $q<0$ are constants which do not depend on $\mu$ and $\nu$.

\section{Application}

We recall that in the construction of an OT-manifold, the group $C:=$ $\mathbb{C}^{m} / \sigma\left(\mathcal{O}_{K}\right)$ is a Cousin group. As a corollary of theorem 4.3, we have:

Corollary 4.4. The Cousin group $C$ satisfies condition $n^{\circ} 2$ of theorem 4.1.

\section{REFERENCES}

1. Y. Abe and K. Kopfermann, Toroidal groups, Lecture Notes in Mathematics, vol. 1759, Springer-Verlag, Berlin, 2001, Line bundles, cohomology and quasi-abelian varieties.

2. F. Berteloot and K. Oeljeklaus, Invariant plurisubharmonic functions and hypersurfaces on semisimple complex Lie groups, Math. Ann. 281 (1988), no. 3, 513-530.

3. P. Cousin, Sur les fonctions triplement périodiques de deux variables, Acta Math. 33 (1910), no. 1, 105-232.

4. N. I. Feldman and Yu. V. Nesterenko, Transcendental numbers, Number theory, IV, Encyclopaedia Math. Sci., vol. 44, Springer, 1998, pp. 1-345.

5. B. Gilligan, C. Miebach, and K. Oeljeklaus, Homogeneous Kähler and Hamiltonian manifolds, Math. Ann. 349 (2011), no. 4, 889-901.

6. A. T. Huckleberry and G. A. Margulis, Invariant analytic hypersurfaces, Inventiones Mathematicae 71 (1983), no. 1, 235-240.

7. K. Oeljeklaus and M. Toma, Non-Kähler compact complex manifolds associated to number fields, Ann. Inst. Fourier (Grenoble) 55 (2005), no. 1, 161-171. 
8. Liviu Ornea and Misha Verbitsky, Oeljeklaus-Toma manifolds admitting no complex subvarieties, Math. Res. Lett. 18 (2011), no. 4, 747-754.

9. C. Vogt, Line bundles on toroidal groups, J. Reine Angew. Math. 335 (1982), 197215.

10. (1983), no. 1-3, 217-232.

LAURENT BATTISTI \& KARL OELJEKLAUS

AIX-MARSEILLE UNIVERSITÉ

CNRS - LATP - UMR 7353

F-13453 MARSEILLE

FRANCE

battisti@cmi.univ-mrs.fr, karloelj@cmi.univ-mrs.fr 